\title{
Benefits of Aerobic Exercise for Breast Cancer Survivors: A Systematic Review of Randomized Controlled Trials
}

\author{
Amira Hassan Bekhet ${ }^{1,2 *}$, Ahmed Ramadan Abdalla ${ }^{3}$, Horeya M Ismail ${ }^{2,4}$, Doaa \\ M Genena ${ }^{5}$, Nermin A Osman ${ }^{5}$, Ayman El Khatib ${ }^{6}$, Rami Labib Abbas ${ }^{6}$
}

\begin{abstract}
Background: Physical exercise may be beneficial to breast cancer (BC) survivors. Here, we systematically summarized the effects of aerobic exercise in BC survivors. We conducted a systematic review of randomized controlled trials (RCTs). Methods: We searched PubMed, Web of knowledge, Scopus, Cochrane Central, Virtual Health Library and PEDRO databases for relevant RCTs, comparing aerobic exercise with usual care among BC survivors. Data were extracted and evidence was synthesized narratively. Results: Twelve studies were included in this systematic review. Studies reported that aerobic exercise can significantly improve the quality of life in BC survivors. Moreover, aerobic exercise alleviated the symptoms of depression and anxiety. However, current evidence from the included studies showed that there was no significant benefit for aerobic exercise in terms of weight loss. Conclusion: Our study suggests that aerobic exercise is beneficial to BC survivors. Clinical Relevance: Aerobic exercise should be recommended in the therapeutic and rehabilitative regimens of $\mathrm{BC}$ survivors.
\end{abstract}

Keywords: Aerobic exercise- breast cancer- physical activity- quality of life

Asian Pac J Cancer Prev, 20 (11), 3197-3209

\section{Introduction}

Breast cancer $(\mathrm{BC})$ is the second most common cancer worldwide and the most frequent cancer among women (25\% of the overall cancer among women) with an increasing incidence in the last decade. The World Health Organization (WHO) ranked $\mathrm{BC}$ as the fifth most common cause of cancer mortality worldwide $(522,000$ deaths in 2012) (Torre et al., 2015). The significant improvements in $\mathrm{BC}$ screening, diagnosis, and treatment over the past few decades led to a decline in BC-related death rate, which in turn caused a substantial increase in the number of BC survivors (Siegel et al., 2017).

Physical activity plays a significant role in improving quality of life (QoL), physical, psychological functions, and other health indicators in cancer patients (Fong et al., 2012). The literature showed several benefits for exercise therapy as a supportive care for BC patients and survivors (Brockow et al., 2016; Kim et al., 2009; McNeely et al., 2006a) These beneficial effects vary according to disease stage, type of primary treatment and the lifestyle of patients (Knols et al., 2005)

The survival outcome in BC survivors is likely to have some correlation with exercise; long-term physical activity levels are important for $\mathrm{BC}$ prognosis and are associated with improved survival (Bertram et al., 2011). Moreover, previous research showed that exercise improves the QoL and lessens the symptoms of depression and anxiety in BC survivors (Segar et al., 1998). Additionally, it was associated with benefits on muscle strength and body composition (Zhu et al., 2016). Therefore, the American Cancer Society guidelines recommend at least 150 minutes on a weekly basis for exercise practicing. These guidelines also encouraged long-term adherence to daily exercise beyond the initial post-surgical rehabilitation (Rock et al., 2012).

Aerobic exercise is a form of physical activity that depends primarily on the aerobic energy-generating process i.e. it requires free oxygen to meet the demands of aerobic metabolism. It involves running/jogging, swimming, cycling, and walking. Several randomized controlled trials (RCTs) suggested the efficacy of aerobic exercise in reducing cancer-related fatigue among $\mathrm{BC}$ survivors (Cantarero-Villanueva et al., 2013; Carter et al., 2016; Vardar Yağlı et al., 2015). Moreover, aerobic physical activity was found effective in improving anthropometric measures as body weight, body composition, and VO2 peak, as well as reducing inflammatory markers (Jones et al., 2013; Matthews et al., 2007; Tizdast et al.,2016)..

The benefits of exercise in general in $\mathrm{BC}$ survivors

${ }^{I}$ Faculty of Physical Therapy, Cairo University, ${ }^{2}$ Medical Research Group of Egypt Cairo, ${ }^{3}$ Faculty of Medicine, Mansoura University, Mansoura, ${ }^{4}$ Cancer Research Cluster, ${ }^{5}$ Medical Research Institute, Alexandria, University, Alexandria, Egypt, ${ }^{6}$ Department of Physical Therapy, Faculty of Health Sciences, Beirut Arab University, Beirut, Lebanon. *For Correspondence: 1300096@st.pt.cu.edu.eg 
have been assessed in several systematic reviews and meta-analyses (Floyd and Moyer, 2010; Meneses-Echávez et al., 2015; Zhu et al., 2016). However, there is a gap in addressing the effects of specific exercise types in $\mathrm{BC}$ rehabilitation program as published trials showed conflicting results, especially in terms of physical activity and QoL outcomes. According to our knowledge, no former systematic review or meta-analysis has assessed the benefits of aerobic exercise specifically in BC survivors. The National Comprehensive Cancer Network (NCCN) reported the need for future research work to identify the effects of specific modes of exercise on specific outcomes as fatigue and QoL in cancer survivors (Schmitz et al., 2010).

We performed this systematic review of the literature to investigate the efficacy of aerobic exercise intervention on physical activity, QoL (primary outcomes), weight, inflammatory markers and sleep among BC survivors by scrutinizing the published trials.

\section{Materials and Methods}

We followed the PRISMA statement guidelines (Table S1 of Supplementary material) during the preparation of this review (Moher et al., 2009). This systematic review is registered on the PROSPERO international prospective register of systematic reviews (CRD42017060106).

\section{Literature search strategy}

We searched PubMed https://www.ncbi.nlm.nih.gov/ pubmed/, Web of knowledge http.apps.webofknowledge. com, Scopus https-www-scopus.com, Cochrane Central https://www.cochranelibrary.com/central, Virtual Health Library (VHL) https://bvsalud.org/en/ and PEDRO https:// www.pedro.org.au/ for RCTs that compared Aerobic Exercise with usual care in BC survivors. We used the following search strategy "(breast cancer survivors* OR breast tumor survivors*) AND (Aerobic exercise OR physical fitness OR physical therapy OR rehabilitation)". No restrictions by publication period were employed.. In addition, we manually scanned the references list of selected articles for relevant studies.

\section{Eligibility criteria and study selection}

We included RCTs that met our inclusion criteria, a) Population: BC survivor women, treated with chemotherapy or radiotherapy (stopped at least six months earlier) or patients who have started adjuvant endocrine therapy (antiestrogens, aromatase inhibitors, LHRH agonists, or combinations) not less than 4 months earlier and not scheduled for and not currently undergoing chemo-/radiation therapy, b) Intervention: Studies in which the intervention was aerobic exercise (walking, cycle ergometers, swimming, and stair climbing), not mixed with any other type of exercise, c) Comparator: Studies where the control group received usual care, d) Outcomes: Physical Activity level, QoL, Sleep parameters, weight, as well as inflammatory markers.

We excluded studies with the following criteria: a) patients on active therapy, b) patients with uncontrolled cardiac or vascular disease, c) patients exercising at a regular basis at baseline, and d) non-English articles. The retrieved citations were added to a Microsoft Excel sheet that was distributed to reviewers. Eligibility screening was done through two separate steps: a) titles and abstracts screening, and b) full texts screening. Each study title and/ or full-text was screened by two independent reviewers (therefore, each reviewer screened half the retrieved citations) and disagreements were resolved by discussion between all reviewers.

\section{Data Extraction}

Three independent reviewers extracted the relevant data from the included studies, using a preformatted data extraction sheet. Disagreements were resolved by the opinion of a fourth reviewer. The extracted data included: a) baseline characteristics of participants as age and cancer stage, b) general characteristics of included studies as design, sample size, and assessed outcomes, c) characteristics of the intervention as type, intensity, and duration and d) the results of each included study. The summary effect size was difficult to calculate due to the lack of sufficient data for subsequent pooling, as well as lack of the homogeneity needed to make a calculation.

\section{Risk of bias assessment}

The risk of bias in included RCTs was assessed according to the Cochrane Handbook for Systematic Reviews of Interventions 5.1.0 (updated March 2011) using the risk of bias assessment table, provided in the same book (part 2, Chapter 8.5). The Cochrane risk of bias assessment tool includes the following domains: Random sequence generation (selection bias), allocation sequence concealment (selection bias), blinding of participants and personnel (performance bias), blinding of outcome assessment (detection bias), incomplete outcome data (attrition bias), selective outcome reporting (reporting bias), and other potential sources of bias. The reviewer judgment is categorized as low risk, high risk, or unclear risk of bias. For risk of bias exclusion across the included studies, we compared the reported outcomes between the studies to exclude reporting of selective outcome.

\section{Results}

\section{Literature search results}

We retrieved 1,800 unique citations. After the initial title and abstract screening, 86 full-text articles were retrieved and screened for eligibility. After full text screening, 12 RCTs ( $n=1,120$ patients) were included in our systematic review (see PRISMA flow diagram; Figure 1).

\section{Characteristic of included studies}

The sample size of included studied ranged from 19 to 357 patients, with an average age of 44 to 61 years. Aerobic exercise was the only intervention of interest in this meta-analysis either supervised or home-based training programs. Most of the included studies reported walking as the preferred type of activity among BC survivors. Exercise sessions were of moderate intensity. Irwin et al., (2008), Matthews et al., (2007) and Saarto 
DOI:10.31557/APJCP.2019.20.11.3197 Aerobic Exercise among Breast Cancer Survivors

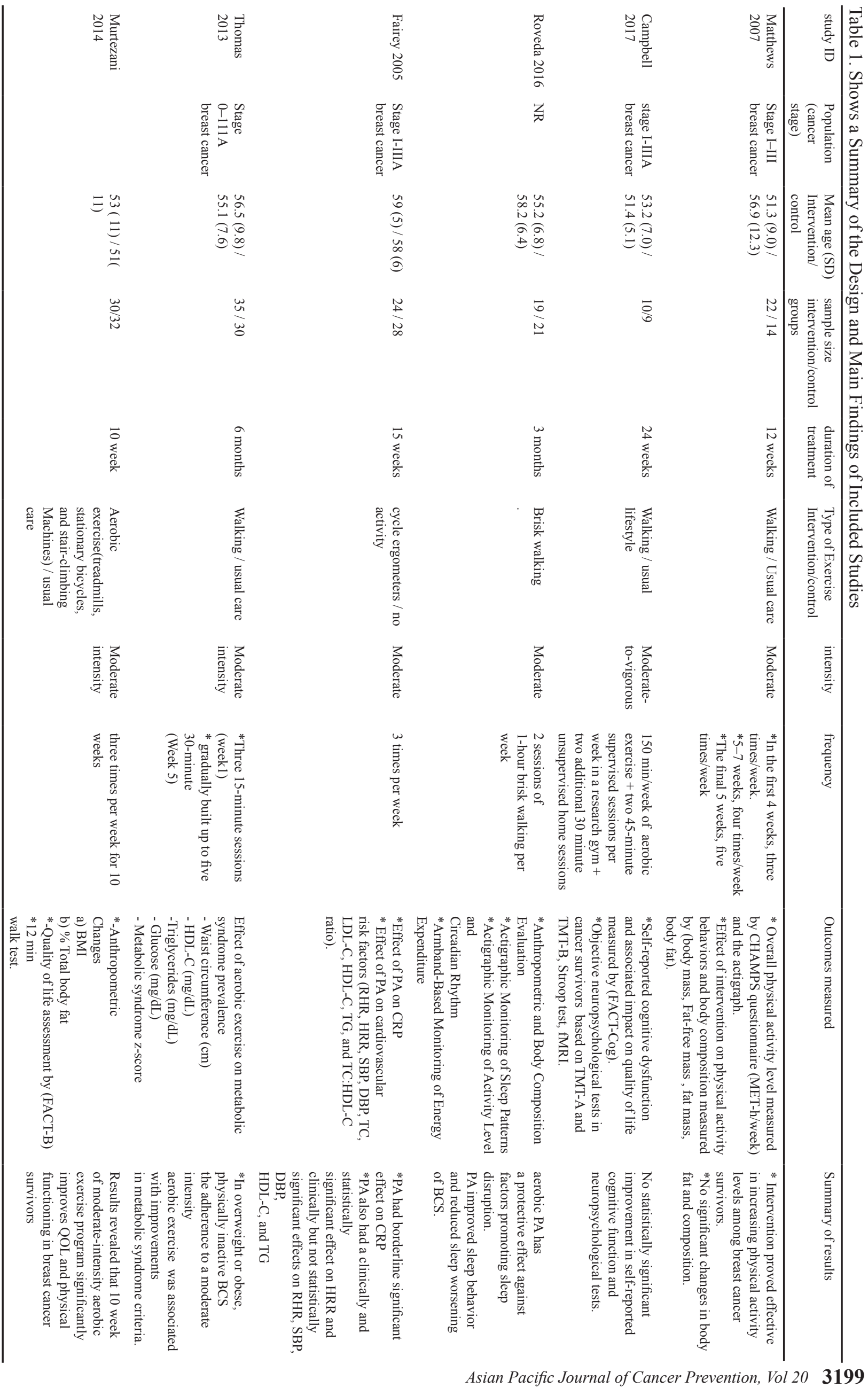




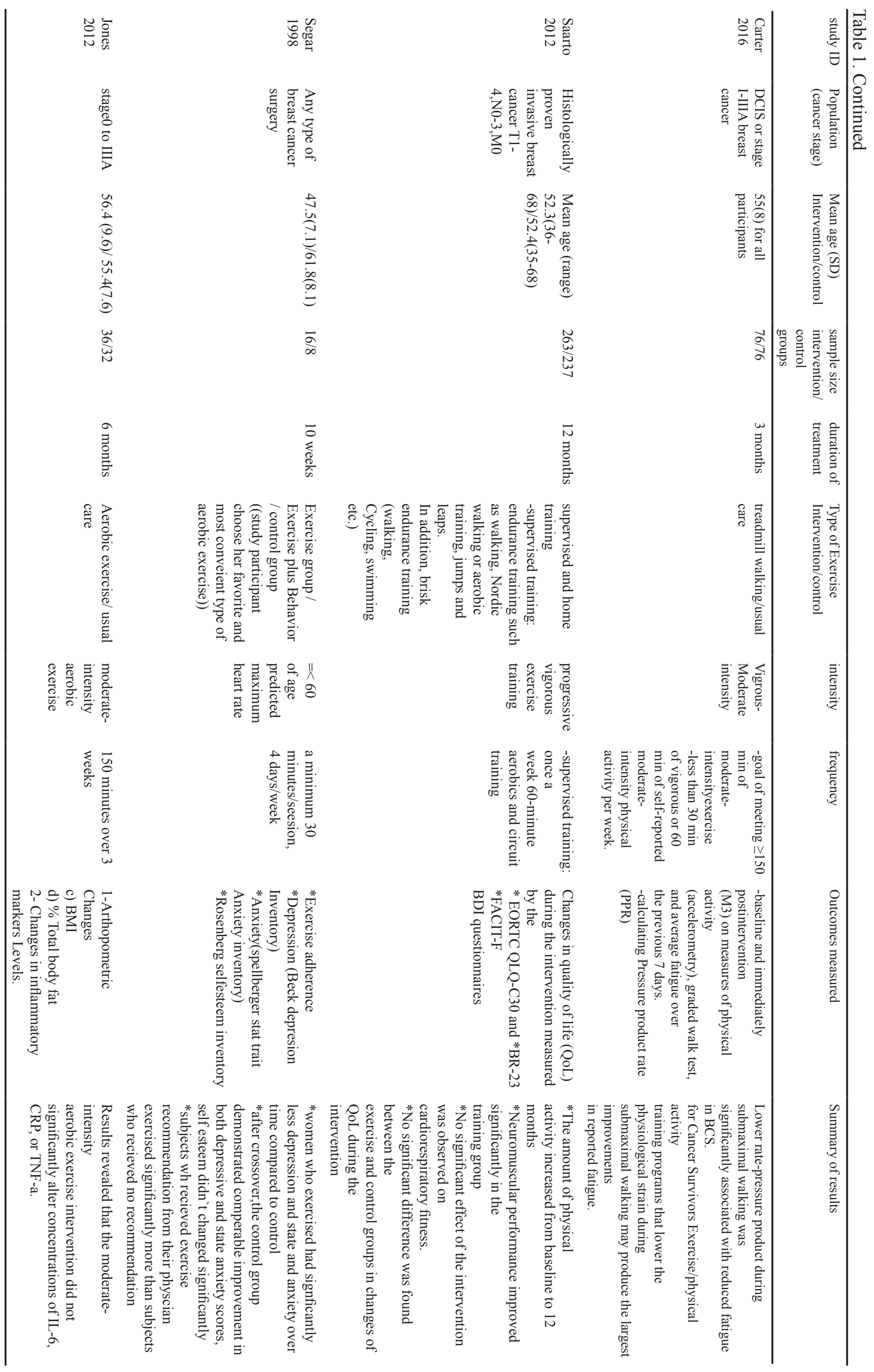

3200 Asian Pacific Journal of Cancer Prevention, Vol 20 
DOI:10.31557/APJCP.2019.20.11.3197

Aerobic Exercise among Breast Cancer Survivors

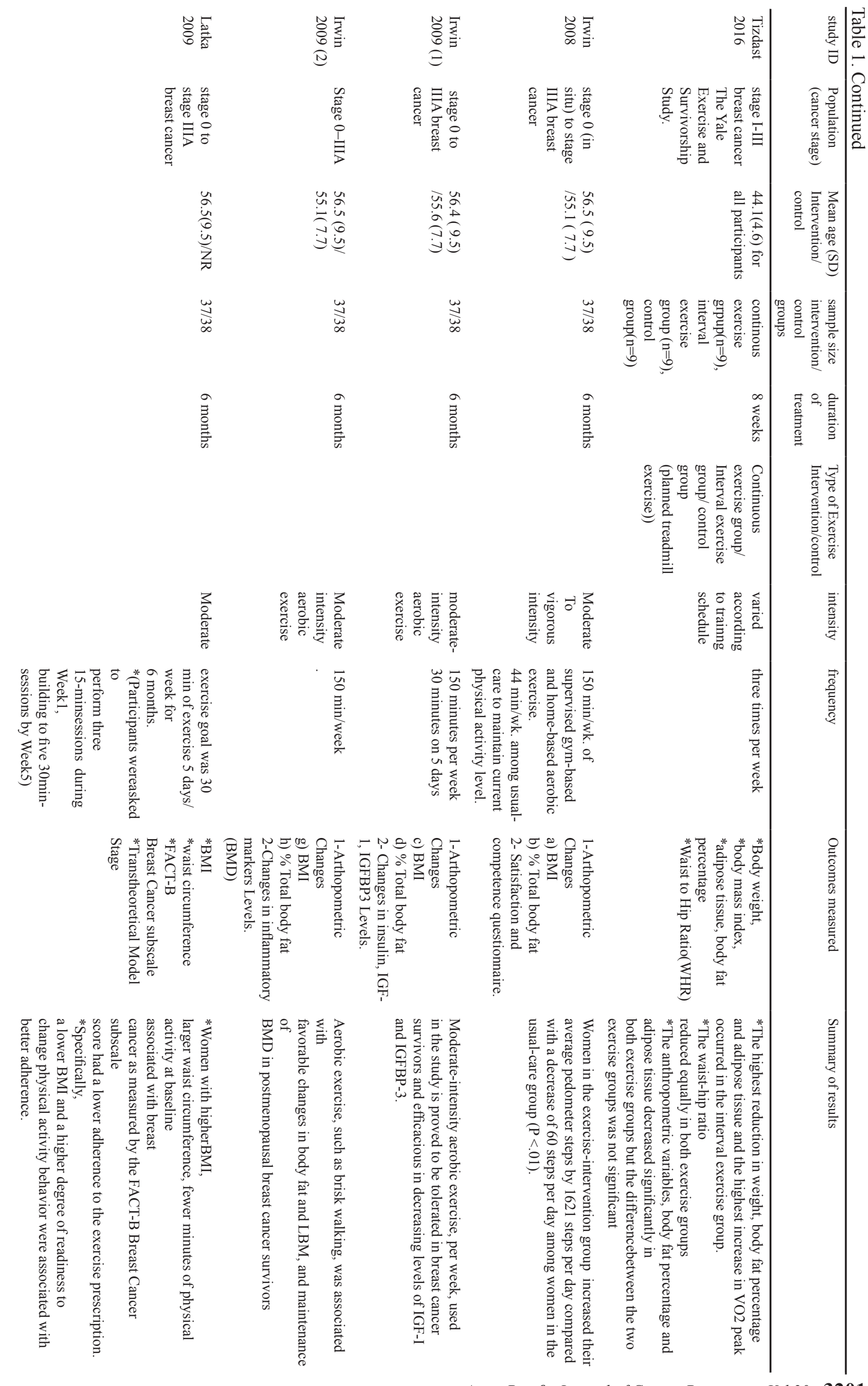




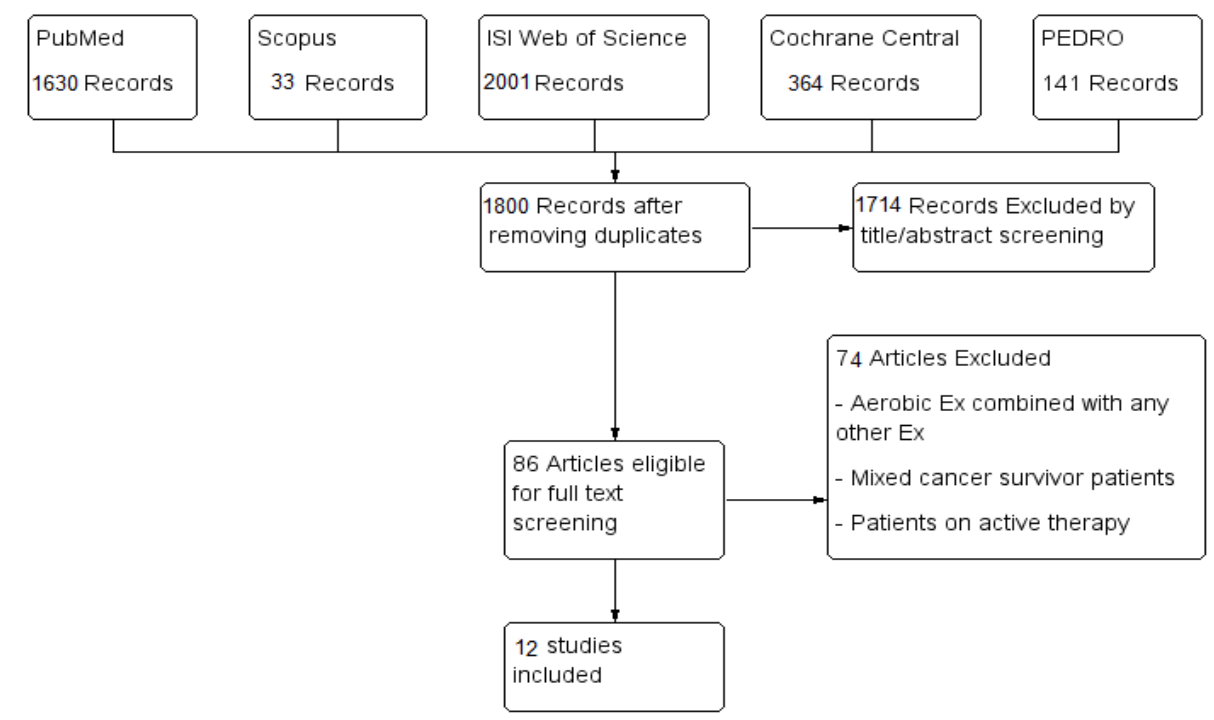

Figure 1. PRISMA Flow Diagram of Screening and Study Selection Process

from fatigue.

\section{Quality of life}

Saarto et al., (2012) measured the QoL using the EORTC-QIQ-C30 or BR-23 forms. They reported no significant difference after 12 months in QoL between

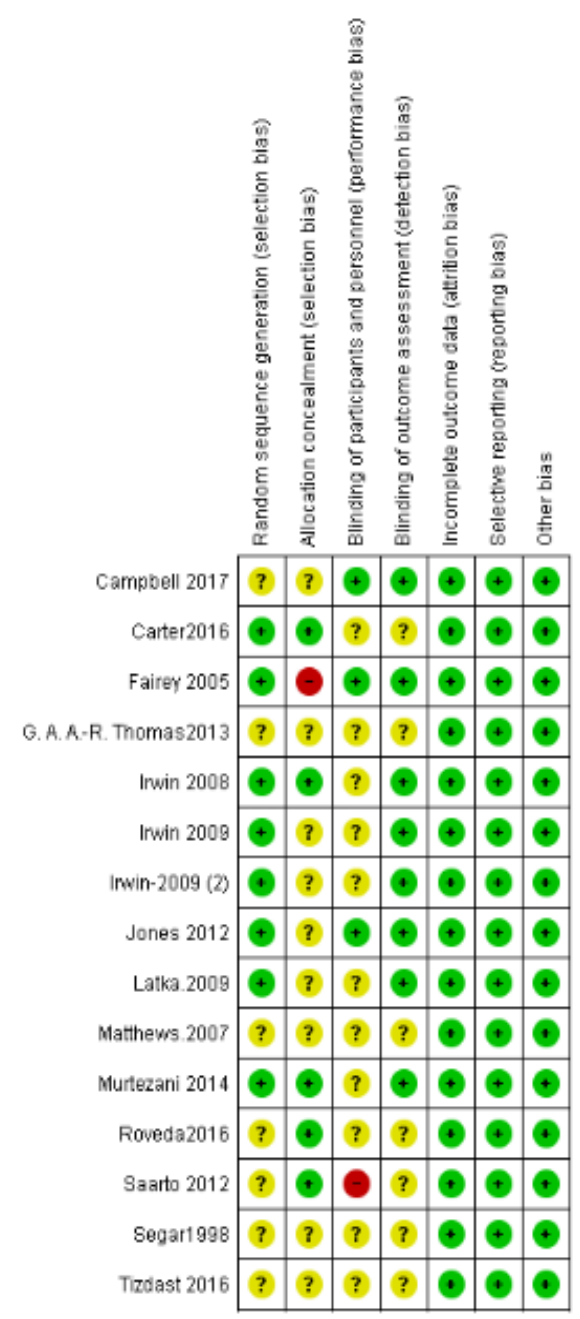

Figure 2. Risk of Bias Summary for Included Studies et al., (2012) assessed physical activity outcome, while Campbell et al., (2017), Murtezani et al., (2014) and Saarto et al., (2012) examined the effect of aerobic exercise on the QoL. Murtezani et al., (2014), Roveda et al., (2016) and Tizdast et al., (2016) investigated the role of aerobic exercise on weight reduction among $\mathrm{BC}$ survivors. Two studies assessed the effect of aerobic exercise on inflammatory markers (Fairey et al., 2005; Jones et al., 2013). Only one study reported the effect of aerobic exercise on sleep parameters (Roveda et al., 2016). A summary of the design and main findings of included studies is shown in Table 1, and the baseline characteristics of their participants are shown in Table 2.

\section{Risk of Bias assessment results}

We used the Cochrane risk of bias assessment tool to assess the bias of included studies. All included studies were of low risk in terms of selective reporting and attrition bias. Only five studies were of low risk of bias in terms of allocation concealment (Carter et al., 2016; Irwin et al., 2008; Murtezani et al., 2014; Roveda et al., 2016; Saarto et al., 2012), while three studies were of low risk of bias in terms of blinding the participants/personnel (Campbell et al., 2017; Fairey et al., 2005; Jones et al., 2013). The summary of risk of bias assessment is shown in Figure 2, and the reasons for authors' judgments are shown in Table S2 of Supplementary material.

\section{Physical Activity}

Mathews et al., (2007) reported higher activity levels in the intervention group compared to usual care group ( $\mathrm{p}$ $\leq 0.04$ ). Moreover, Irwin et al., (2008) reported that in the 7-day physical activity log, the exercise group increased from moderate to vigorous intensity reactional activity (129 min per week, $p<0.001$ ), compared to a smaller increase in the usual care group (45 min per week). However, Saarto et al., (2012) reported an insignificant difference between the exercise and control groups $(\mathrm{P}=0.97)$. They detected a significant linear trend between higher physical activity, improved QoL, and recovery 
DOI: 10.31557/APJCP.2019.20.11.3197

Aerobic Exercise among Breast Cancer Survivors

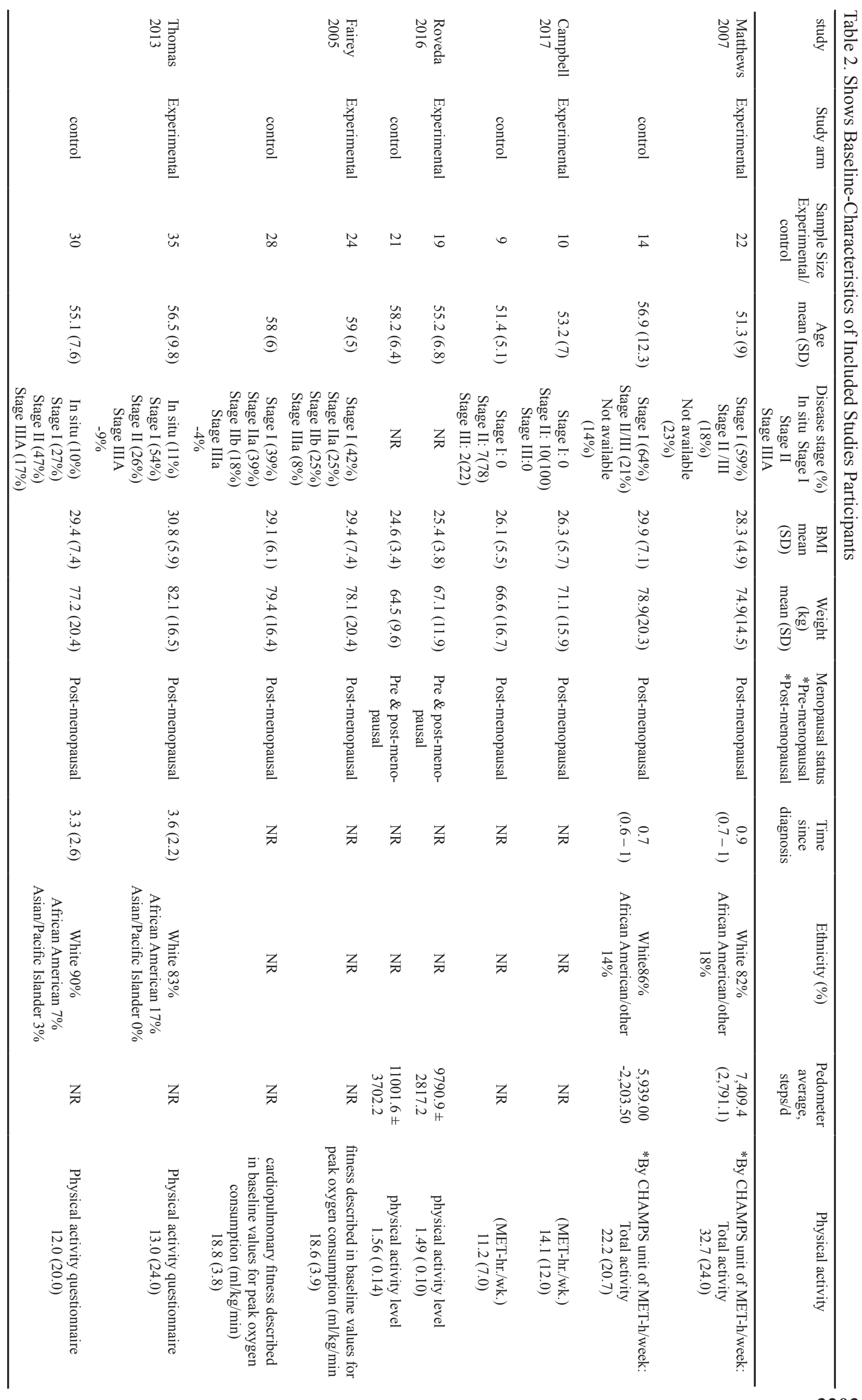




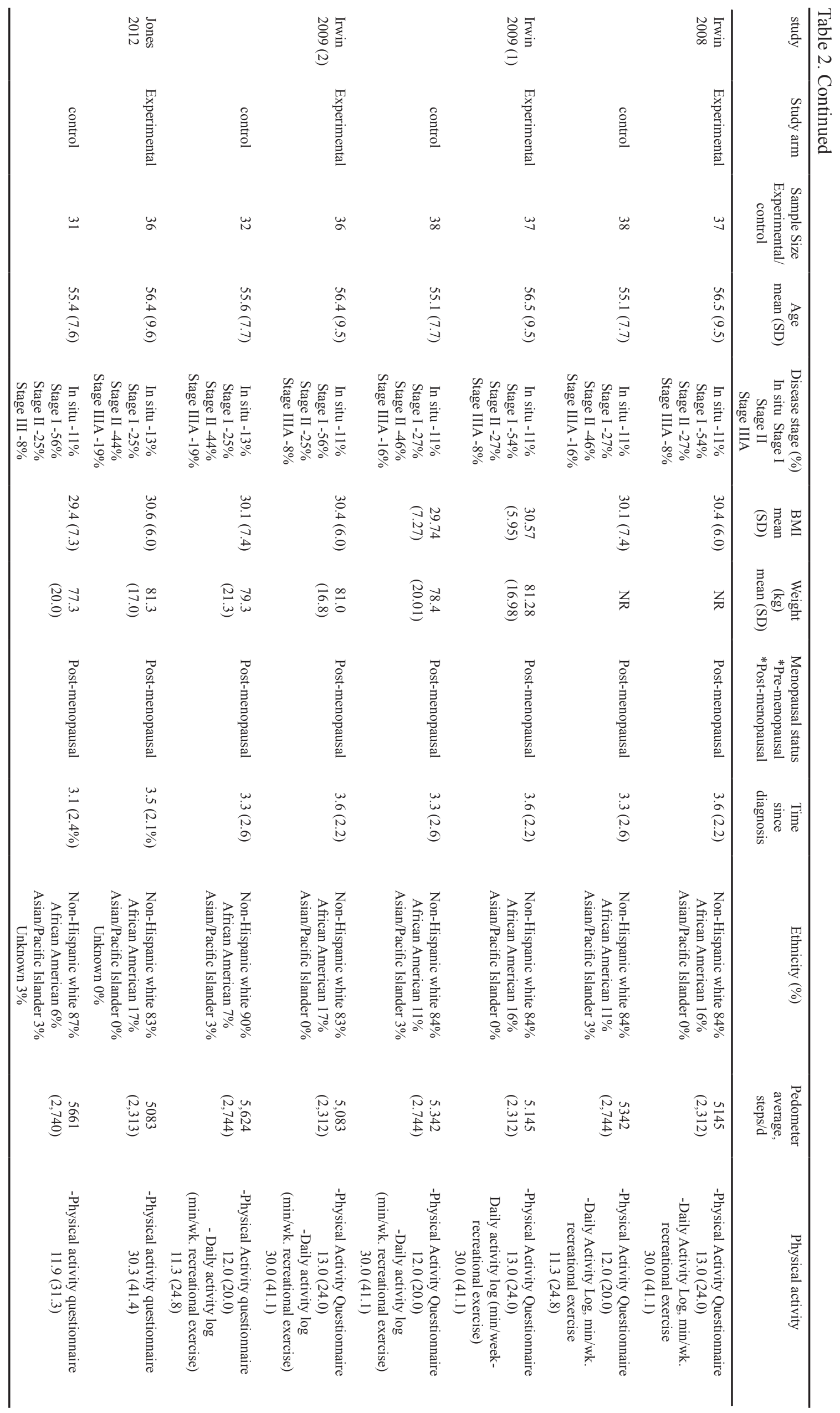


DOI: 10.31557/APJCP.2019.20.11.3197

Aerobic Exercise among Breast Cancer Survivors

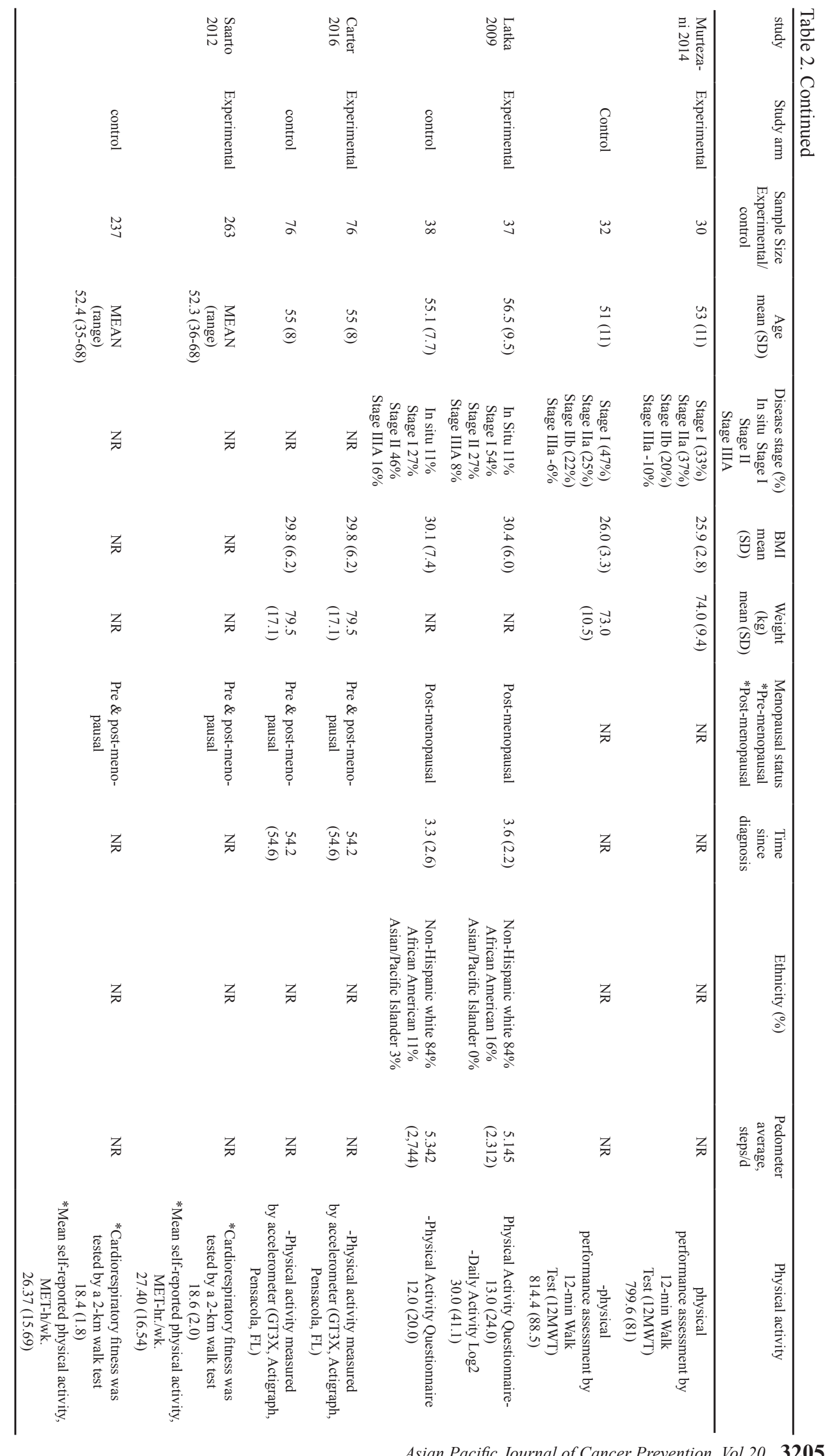




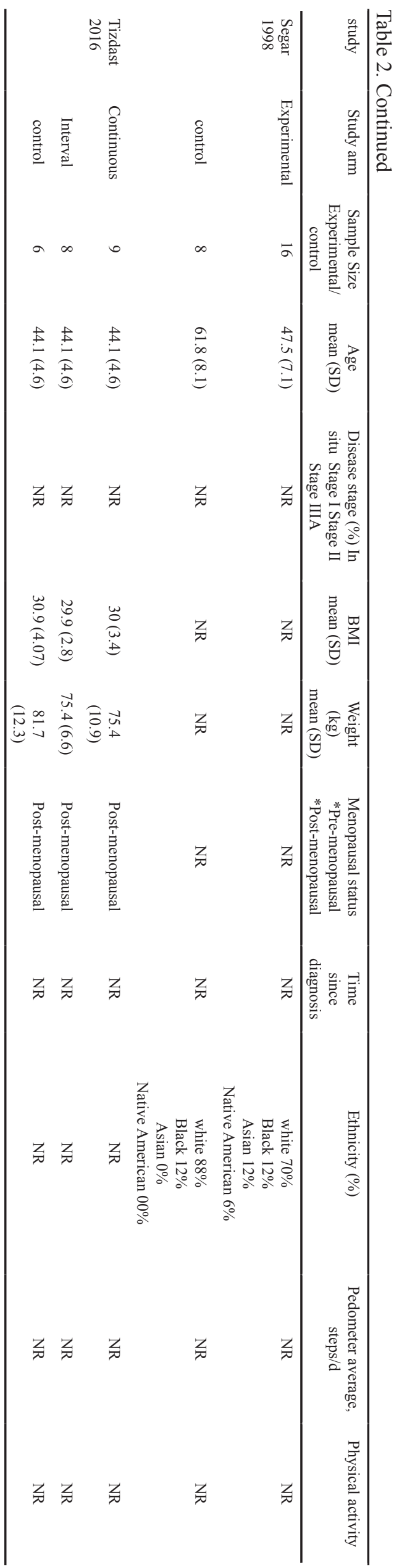

exercise and control groups. Campbell et al., (2017) used the FACT-COG tool to measure the cognitive symptoms impacting the QoL, but they found no statistically significant improvement. Murtezani et al., (2014) used the FACT B score to measure the overall QoL after 10 weeks of intervention. They reported that the FACT B score was increased in the intervention group and was decreased in controls $(\mathrm{P}=0.003)$.

\section{Weight}

Tizdast et al., (2016) reported that higher weight reduction was achieved in the interval exercise group ( $\mathrm{P}=0.012$ ). Murtezani et al., (2014) and Fairy et al., (2005) did not observe any significant difference between the exercise and control groups in terms of weight loss ( $95 \% \mathrm{CI},-4.8$ to $2.25 \mathrm{Kg}$; $\mathrm{P}=0.47$ and $95 \% \mathrm{CI},-1.6$ to 0.6 $\mathrm{Kg} ; \mathrm{P}=0.39$, respectively). On the contrary, Roveda et al., (2016) found that the control group had a significant weight reduction after 3 months of physical activity $(\mathrm{P}=0.01)$.

\section{Inflammatory markers}

Two studies examined the effects of aerobic exercise on inflammatory makers in BC survivors. Fairy et al., (2005) reported that C-reactive protein (CRP) decreased by $139 \mathrm{mg} / \mathrm{l}$ in the exercise group, while it increased by $0.1 \mathrm{mg} / \mathrm{l}$ in the control group. However, this change was not significant ( $95 \% \mathrm{CI},-3.09$ to $0.1 \mathrm{mg} / \mathrm{l} ; \mathrm{P}=0.66$ ) Moreover, Jones et al. (2013) reported an insignificant difference between randomization groups regarding serum concentrations of interleukin (IL)-6, CRP, and tumor necrosis factor $(\mathrm{TNF}-\alpha)$.

\section{Sleep parameters}

Only one study reported the effects of exercise on sleep parameters for BC survivors. Roveda et al., (2016) found sleep efficiency (SE) to be significantly decreased in the control group, it but remained unchanged in the exercise group $(\mathrm{P}=0.001)$. The mean activity score (MAS) was significantly increased in the control group, but it remained unchanged in the exercise group $(\mathrm{p}<0.001)$. The authors reported a significant decrease $(\mathrm{P}=0.03)$ in the actual sleep time (AST) and a significant increase $(\mathrm{P}=0.02)$ in the movement fragmentation index (MFI) after aerobic exercise intervention, compared to baseline scores.

\section{Discussion}

This systematic review summarized the evidence on the effects of aerobic exercise interventions (especially walking as the most frequently investigated approach) in BC survivor women. The results showed that aerobic exercise was superior to usual care in enhancing the physical activity, QoL, and sleep parameters. However, weight and inflammatory markers did not show any remarkable difference between the two groups.

Moderate intensity aerobic exercise is associated with increased short-term physical activity levels among BC survivors, especially in patients who did not follow regular exercise before (Irwin et al., 2008; Matthews et al., 2007). 
However, Saarto et al., (2012) reported no significant difference between the exercise and control groups. This is explained by the considerable motivation and healthy lifestyle in the control group patients, which exerted a ceiling effect on the ability to detect the exercise benefits.

The observed increase in physical activity levels significally correlates with QoL improvement. The results of Murtezani et al., (2014) confirmed the causative relationship between moderate intensity aerobic exercise and QoL improvement. These results are supported by previous studies on the efficacy of aerobic exercise in improving the overall QoL (Burnham., 2002; McNeely et al., 2006). This improvement may be due to reducing anxiety and increasing energy in addition to counteracting some negative effects of cancer treatment (Jones et al., 2013; Meneses-Echávez et al., 2015; Murtezani et al., 2014).

Campbell et al., (2017) found that aerobic exercise ameliorated the resulting cognitive impairment from the psychological burden and cancer therapies. The results of this study come in accordance with a previous meta-analysis of $33 \mathrm{RCTs}$, which investigated the effects of exercise in general regarding the psychological outcomes, body composition and physical fuctions (Zhu et al., 2016). It concluded that exercise was beneficial for BC survivors because it improved the QoL, body configuration, and muscle strength, decreased serum levels of insulin, IGF-II, and IGFBP-1, and alleviated depression and anxiety.

Roveda et al., (2016) examined the effects of exercise on the sleep pattern. They reported significant improvement of sleep disturbance among $\mathrm{BC}$ survivors. Another study conducted by Thomas et al., (2013) revealed positive effects for aerobic exercise on reversing the metabolic syndrome in sedentry BC survivors. Another study examined the effect of aerobic exercise in untrained women in a meta-analysis framework. It included different age groups and exercise intensities and emphasized the golden role of early exercise intervention in improving the cardiovascular outcomes in sedentary women (Zhang et al., 2017).

\section{Strengths of the study}

We followed the PRISMA statement during performance and reporting of this review and conducted all steps in accordance to the cochrane handbook for systematic reviews of interventions. Reasons for patient discontinuation were appropriately addressed. Moreover, study investigators used as intention to treat approach so there would not be attrition bias. Studies included in this systematic review were low to moderate risk of bais according to Cochrane ROB assessment tool.

\section{Limitations of the study}

We selectively chose studies that investigated the benefits of aerobic exercise in BC survivors. We found scarce literature covering this pattern of exercise with diversity in the measured outcomes, intervention (type, intensity, and duration), and the used assessment methods. Therefor, the extracted data were not sufficient for subsequent pooled analysis. Future studies are recommended to study the effects of aerobic exercise in $\mathrm{BC}$ survivors for longer durations with including much important outcomes, such as inflammatory markers, sleep pattern, and other cancer contol measures.

\section{Clinical Implications}

The review findings supports that aerobic exercise intervention is beneficial to $\mathrm{BC}$ survivor women. It improves fatigue associated with $\mathrm{BC}$ by improving levels of physical activity, as well as QoL. It also enhances the stability of sleep among BC survivors (Matthews et al., 2007; Murtezani et al., 2014; Roveda et al., 2016).

\section{Key Practice Points}

* Aerobic exercise should be recommended in the rehbailitation regimen of $\mathrm{BC}$ survivors.

* The intensity and duration of exercise should be personalized to each patient and further research is needed in this regard.

In conclusion, despite the aforementioned limitations, there is evidence that aerobic exercise (mainly in the form of walking) was associated with beneficial outcomes in $\mathrm{BC}$ survivor women; It improved the Qol and alleviated the symptoms of depression and anxiety. Moreover, it enhanced the physical functioning and sleep pattern.

\section{Abbreviations}

BC: Breast Cancer, CRP: C - reactive protein, QoL: Quality of Life.

\section{References}

Burnham TR, Wilcox A (2002). Effects of exercise on physiological and psychological variables in cancer survivors. Med Sci Sports Exerc, 34, 1863-7.

Campbell KL, Kam JWY, Neil-Sztramko SE, et al (2017). Effect of aerobic exercise on cancer-associated cognitive impairment: A proof-of-concept RCT. Psychooncology, https://doi.org/10.1002/pon.4370.

Cantarero-Villanueva I, Fernandez-Lao C, Cuesta-Vargas AI, et al (2013). The effectiveness of a deep water aquatic exercise program in cancer-related fatigue in breast cancer survivors: A randomized controlled trial. Arch Phys Med Rehab, 94, 221-30.

Carter SJ, Hunter GR, McAuley E, et al (2016). Lower rate-pressure product during submaximal walking: a link to fatigue improvement following a physical activity intervention among breast cancer survivors. J Cancer Surviv, 10, 927-34.

Cooper JM, Loeb SJ, Smith CA, et al (2010). The primary care nurse practitioner and cancer survivorship care. $\mathrm{J} \mathrm{Am} \mathrm{Acad}$ Nurse Pract, 22, 394-402.

Dieli-Conwright CM, Lee K, Kiwata JL, et al (2016). Reducing the risk of breast cancer Recurrence: an Evaluation of the Effects and Mechanisms of diet and exercise. Curr Breast Cancer Rep, 8, 139-50.

Fairey AS, Courneya KS, Field CJ, et al (2005). Effect of exercise training on C-reactive protein in postmenopausal breast cancer survivors: A randomized controlled trial. Brain Behav Immun, 19, 381-8.

Floyd A, Moyer A (2010). Group versus individual exercise interventions for women with breast cancer: A meta-analysis. Health Psychol Rev, 4, 22-41.

Fong DY, Ho JW, Hui BP, et al (2012). Physical activity for 
cancer survivors: meta-analysis of randomised controlled trials. $B M J, 344,70$.

Hall-Alston J (2015). Exercise and the breast cancer survivor: The role of the nurse practitioner. Clin J Oncol Nurs, 19, 98-102.

Irwin ML, Cadmus L, Alvarez-Reeves M, et al (2008). Recruiting and retaining breast cancer survivors into a randomized controlled exercise trial: The Yale Exercise and Survivorship Study. Cancer, 112, 2593-2606.

Jones LW, Alfano CM (2013). Exercise-oncology research: Past, present, and future. Acta Oncol, 52, 195-215.

Jones SB, Thomas GA, Hesselsweet SD, et al (2013). Effect of exercise on markers of inflammation in breast cancer survivors: The yale exercise and survivorship study. Cancer Prev Res, 6, 109-118.

Kim CJ, Kang DH, Park JW (2009). A meta-analysis of aerobic exercise interventions for women with breast cancer. West J Nurs Res, 31, 437-61.

Knols R, Aaronson NK, Uebelhart D, Fransen J, Aufdemkampe G (2005). Physical exercise in cancer patients during and after medical treatment: A systematic review of randomized and controlled clinical trials. J Clin Oncol, 23, 3830-42.

Ligibel J (2012). Lifestyle factors in cancer survivorship. J Clin Oncol, 30, 3697-704.

Markes M, Brockow T, Resch KL (2006). Exercise for women receiving adjuvant therapy for breast cancer. https://doi. org/10.1002/14651858.CD005001.pub2

Matthews CE, Wilcox S, Hanby CL, et al (2007). Evaluation of a 12-week home-based walking intervention for breast cancer survivors. Support Care Cancer, 15, 203-11.

McNeely ML, Campbell KL, Rowe BH, et al (2006a). Effects of exercise on breast cancer patients and survivors: a systematic review and meta-analysis. CMAJ, 175, 34-41.

McNeely ML, Campbell KL, Rowe BH, et al (2006b). Effects of exercise on breast cancer patients and survivors: a systematic review and meta-analysis. CMAJ, 175, 34-41.

Meneses-Echávez JF, González-Jiménez E, Ramírez-Vélez R (2015). Effects of supervised exercise on cancer-related fatigue in breast cancer survivors: A systematic review and meta-analysis. BMC Cancer, 15, 1-13.

Moher D, Liberati A, Tetzlaff J, et al (2009). Preferred reporting items for systematic reviews and meta-analyses: The PRISMA statement. PLoS Med, Public Library of Science. https://doi.org/10.1371/journal.pmed.1000097.

Murtezani A, Ibraimi Z, Bakalli A, et al (2014). The effect of aerobic exercise on quality of life among breast cancer survivors: A randomized controlled trial. $J$ Cancer Res Ther, 10, 658-64.

Rock CL, Doyle C, Demark-Wahnefried W, et al (2012). Nutrition and physical activity guidelines for cancer survivors. CA Cancer J Clin, 62, 243-74.

Roveda E, Vitale JA, Bruno E, et al (2016). Protective effect of Aerobic physical activity on sleep behavior in breast cancer survivors. Integr Cancer Ther, 1534735416651719-. https:// doi.org/10.1177/1534735416651719.

Saarto T, Penttinen HM, Sievänen H, et al (2012). Effectiveness of a 12-month exercise program on physical performance and quality of life of breast cancer survivors. Anticancer Res, 32, 3875-84.

Schmitz KH, Courneya KS, Matthews C, et al (2010). American college of sports medicine roundtable on exercise guidelines for cancer survivors. Med Sci Sports Exerc, 42, 1409-26.

Segar ML, Katch VL, Roth RS, et al (1998). The effect of aerobic exercise on self-esteem and depressive and anxiety symptoms among breast cancer survivors. Oncol Nurs Forum, 25, 107.

Siegel RL, Miller KD, Jemal A (2017). Cancer statistics, 2017.
CA Cancer J Clin, 67, 7-30.

Thomas GA, Alvarez-Reeves M, Lu L, Yu H, Irwin ML (2013). Effect of exercise on metabolic syndrome variables in breast cancer survivors. Int J Endocrinol, 2013. https://doi. org/10.1155/2013/168797.

Tizdast N, Ghazalian F, Gholami M (2016). The effect of exercise type on inflammatory markers in obese survivors with breast cancer: Randomized Control Trial. Health Scope, 5, https:// doi.org/10.17795/jhealthscope-33421.Research

Torre LA, Bray F, Siegel RL, et al (2015). Global cancer statistics, 2012. CA Cancer J Clin, 65, 87-108.

Vardar Yağlı N, Şener G, Arıkan H, et al (2015). Do yoga and aerobic exercise training have impact on functional capacity, fatigue, peripheral muscle strength, and quality of life in breast cancer survivors?. Integr Cancer Ther, 14, 125-32.

Zhang Y, Xu L, Zhang X, et al (2017). Effects of different durations of aerobic exercise intervention on the cardiovascular health in untrained women: a meta-analysis and meta-regression. J Sport Med Phys Fit, https://doi.org/10.23736/S00224707.17.07029-3.

Zhu G, Zhang X, Wang Y, et al (2016). Effects of exercise intervention in breast cancer survivors: A meta-analysis of 33 randomized controlled trails. Onco Targets Ther, 9, 2153-68.

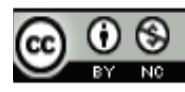

This work is licensed under a Creative Commons AttributionNon Commercial 4.0 International License. 